\title{
Divalproex ER Combined with Olanzapine or Risperidone for Treatment of Acute Exacerbations of Schizophrenia
}

\author{
Daniel E Casey,', David G Daniel 2,3,9, Carol Tamminga ${ }^{4}$, John M Kane', Tram Tran-Johnson ${ }^{6}$, \\ Patricia Wozniak $^{7,8}$, Walid Abi-Saab ${ }^{7}$, Jeff Baker $^{7}$, Laura Redden ${ }^{7}$, Nicholas Greco ${ }^{7}$ and Mario Saltarelli ${ }^{7}$ \\ 'Oregon Health and Science University, Portland, OR, USA; ' George Washington University, Washington, DC, USA; 'Bioniche Development, Falls \\ Church, VA, USA; ${ }^{4}$ University of Texas Southwestern Medical Center, Dallas, TX, USA; ${ }^{5}$ The Zucker Hillside Hospital, Glen Oaks, NY, USA; \\ ${ }^{6}$ California Neuropsychopharmacology Clinical Research Institute, San Diego, CA, USA; ${ }^{7}$ Abbott Laboratories, Abbott Park, IL, USA; ${ }^{8}$ Advanced \\ Clinical Research Services, Bannockburn, IL, USA
}

\begin{abstract}
The objective of this study was to evaluate the efficacy and safety of divalproex sodium extended release (divalproex ER) vs placebo in combination with olanzapine or risperidone for the treatment of acute exacerbations of schizophrenia. In this 12-week, randomized, double-blind, parallel-group, multi-center trial, a total of 402 patients were randomized and treated; 103 received olanzapine/placebo, 99 received olanzapine/divalproex $\mathrm{ER}, \mathrm{I} 0 \mathrm{I}$ received risperidone/placebo, and 99 received risperidone/divalproex ER. Divalproex $\mathrm{ER}$ was initiated on day I at $20 \mathrm{mg} / \mathrm{kg}$ per day q AM and was titrated to clinical effect on days 3, 7, and I0, not to exceed a maximum dosage of $35 \mathrm{mg} / \mathrm{kg}$ per day. Olanzapine and risperidone were initiated at 5 and $2 \mathrm{mg} /$ day q PM, respectively, increased to 10 and $4 \mathrm{mg} /$ day on day 3 , and increased to fixed target doses of 15 and $6 \mathrm{mg} /$ day on day 6 . No significant treatment difference was demonstrated between the combination therapy and antipsychotic monotherapy groups on the primary efficacy variable of the mean change from baseline to day 14 last observation carried forward on the Positive and Negative Syndrome Scale (PANSS) total score, although antipsychotic monotherapy did demonstrate superiority to combination therapy on the PANSS Negative subscale at several time points. Combination therapy also failed to show an advantage over antipsychotic monotherapy at day 84 on the PANSS total score. Most adverse events observed in the study were mild to moderate in severity, and the overall number of adverse events did not differ significantly between the combination therapy groups and their corresponding antipsychotic monotherapy group.

Neuropsychopharmacology (2009) 34, 1330-1338; doi:I0. I038/npp.2008.209; published online 3 December 2008
\end{abstract}

Keywords: divalproex sodium ER; schizophrenia; atypical antipsychotic; psychosis; risperidone; olanzapine

\section{INTRODUCTION}

Schizophrenia is a severe, debilitating, and chronic mental disorder characterized by positive (hallucinations, delusions) and negative (withdrawal, apathy, anhedonia) symptoms. The conventional antipsychotic drugs used to treat schizophrenia are believed to produce their effects through blockade of the dopamine type 2-receptor and are effective in treating positive symptoms but are associated with extrapyramidal side effects (EPS). The second generation antipsychotic drugs (such as risperidone and olanzapine) cause fewer EPS, and have shown promise in reducing the negative symptoms of schizophrenia, possibly due to their inhibition of serotonin type-2 receptor activity (Möller, 2003). In spite of these advances, the treatment of

*Correspondence: Dr DE Casey, Oregon Health and Science University, GHI 249 Department of Psychiatry, 318I S.W. Sam Jackson Park Road, Portland, OR 97239, USA, Tel: + 503418 I29 I, Fax: + 503 4I 8 1294, E-mail: caseyd@ohsu.edu

${ }^{9}$ Current address: United BioSource Corporation, McLean, VA, USA Received 10 July 2008; revised 6 October 2008; accepted 21 October 2008 schizophrenia remains suboptimal; the disorder usually follows a chronic relapsing course, and the side effects of current therapies are of concern (Meltzer, 1999).

Although the monoamines (especially dopamine) have been the major focus of research in the pathophysiology of schizophrenia for many years, evidence also indicates abnormalities in other neurotransmitter systems, including the GABAergic system (Benes, 2000; Lewis et al, 2005). Studies have demonstrated a loss of inhibitory GABAergic neurons in the brains of schizophrenic patients. These findings produced speculation that drugs that enhance GABAergic activity may provide benefit in the treatment of schizophrenia (Benes, 2000; Berle and Spigset, 2005; Lewis et al, 2005).

Divalproex sodium (divalproex hereafter) has putative GABA agonism via multiple mechanisms (reviewed by Wassef et al, 2003) and potentiates antipsychotic-induced dopamine release in the prefrontal cortex of rats (Melzer et al, 2001). A number of small, open-label studies support the clinical benefit of divalproex in combination with conventional antipsychotic medications for the treatment of psychotic symptoms (Gunderewa et al, 1980; Moringo et al, 
1989; Wassef et al, 1989, 2000; Chong et al, 1998). Recently, a large, double-blind, multi-center trial demonstrated improvement in psychotic symptoms for divalproex $v s$ placebo when given in combination with olanzapine or risperidone for the treatment of acute exacerbations of schizophrenia. Improvement with divalproex was seen as early as day 3 and continued to day 21 (Casey et al, 2003).

The objective of this study was to evaluate the effects of divalproex sodium extended release (divalproex ER hereafter) in combination with olanzapine or risperidone $v s$ antipsychotic monotherapy with olanzapine or risperidone for the treatment of schizophrenia over a 12-week period.

\section{METHODS}

\section{Patients}

Hospitalized patients between the ages of 18 and 65 years with an acute exacerbation of schizophrenia were enrolled. Patients with a current DSM-IV-TR diagnosis of schizophrenia, as confirmed by a Structured Clinical Interview for DSM-IV-TR (SCID; First et al, 2002) were selected for inclusion on the basis of having (1) a Positive and Negative Syndrome Scale (PANSS; Kay et al, 1987) total score $\geqslant 70$ (based on a 1-7-point scale for each measure), (2) a score totaling $\geqslant 8$ on any two of the four items from the psychosis cluster of the Brief Psychiatric Rating Scale-derived (BPRSd), and (3) a total score $\geqslant 6$ on either hostility and uncooperativeness or excitement and tension items from the BPRSd. For inclusion, a patient must have had, in the investigator's opinion, a positive response to treatment with antipsychotics within the previous 2 years.

Patients were excluded from the study if they had a current diagnosis of schizoaffective disorder, drug-induced psychosis, manic episode, or major depressive episode, as were those who had serious violent, homicidal, or suicidal ideation, or had more than three psychiatric hospitalizations in the previous 6 months or more than 8 weeks of psychiatric hospitalization in the previous 12 months.

\section{Study Design}

The study was a randomized, double-blind, parallel-group, multi-center trial, consisting of a 1- to 5-day screening/ washout period and a 12-week double-blind treatment period. The protocol was approved by the institutional review board of each participating study site. Written informed consent was obtained from each patient or the patient's authorized representative before enrollment in the study.

Patients were randomized to one of four treatment groups: (1) olanzapine/placebo, (2) risperidone/placebo, (3) olanzapine/divalproex ER, or (4) risperidone/divalproex ER. Divalproex ER was initiated on day 1 at $20 \mathrm{mg} / \mathrm{kg}$ per day $\mathrm{q} A M$ and was titrated to clinical effect on days 3,7 , and 10 , not to exceed a maximum dosage of $35 \mathrm{mg} / \mathrm{kg}$ per day. Olanzapine and risperidone were initiated at 5 and $2 \mathrm{mg} /$ day $\mathrm{q} P M$ respectively, increased to 10 and $4 \mathrm{mg} /$ day on day 3 , and increased to fixed target doses of 15 and $6 \mathrm{mg} /$ day on day 6 . Once these doses were achieved, they were continued for the remainder of the study.
Lorazepam, propranolol hydrochloride, and benztropine mesylate could be used as adjunctive medications, but were not to be used prophylactically. Lorazepam (up to $6 \mathrm{mg} / \mathrm{day}$ during screening, up to $4 \mathrm{mg} / \mathrm{day}$ on days $1-7$ of the treatment period, and up to $2 \mathrm{mg} /$ day from day 8 until hospital discharge) could be prescribed for severe agitation and for control of insomnia, but not within $8 \mathrm{~h}$ before efficacy ratings. Propranolol hydrochloride (according to the investigator's discretion) could be prescribed for severe or intolerable akathisia. Benztropine mesylate (up to $4 \mathrm{mg}$ / day throughout the study) could be prescribed for severe or intolerable akathisia, EPS, or dystonia.

The double-blind treatment period consisted of a 14-day acute phase followed by a 10-week stabilization phase. Patients were required to remain hospitalized for a minimum of 14 days during the Acute Phase of the study and could be discharged (per investigator discretion) anytime after day 14 . Once discharged, patients were seen as outpatients for the remainder of the study and were required to return to the study site for regularly scheduled assessments.

\section{Clinical Evaluations}

The psychiatric status of patients was evaluated using the PANSS total and subscales, and the Clinician's Global Impression (CGI) Severity and Improvement scales (Guy, 1976). The evaluations were conducted on days 1 (baseline), $3,5,7,10,14,21,28,35,42,49,56,63,70,77$, and 84 . The PANSS was scored based on the previous $48 \mathrm{~h}$ symptoms. The BPRSd was administered as a secondary efficacy measure.

All raters received extensive training on rating the PANSS and other key scales, including initial training sessions in which raters were required to rate scales and score within 1.5 standard deviations of the mean of all raters. At approximately midstudy, raters attended refresher training sessions, during which rater scores demonstrated good reliability. Only individuals who successfully completed a rater-training program were permitted to administer and score the rating scales. If at all possible, the same rater performed all ratings for a given subject throughout the study.

\section{Safety Assessment}

Safety assessments included a physical examination, vital sign monitoring, body weight measurement, adverse event collection, and laboratory tests. EPS side effects were evaluated during the double-blind treatment period using the Simpson-Angus Scale (SAS; Simpson and Angus, 1970), the Barnes Akathisia Scale (BAS; Barnes, 1989), and the Abnormal Involuntary Movement Scale (AIMS; Guy, 1976).

\section{Statistical Analysis}

All statistical tests were two tailed with a significance level of 0.05 . The primary treatment comparison for demographic and baseline characteristics and for efficacy endpoints was combination therapy $v s$ antipsychotic monotherapy. The combination therapy group consisted of patients from the olanzapine/divalproex ER and risper- 
idone/divalproex ER groups, and the monotherapy group consisted of patients from the olanzapine/placebo and risperidone/placebo treatment groups. Comparability of treatment groups was assessed by a one-way analysis of variance (ANOVA) for age and weight and by a Wilcoxon rank sum test for age at first diagnosis of schizophrenia. Treatment group differences for lifetime number of hospitalizations and number of suicide attempts (collected as $0,1-5,6-10,11-15,16-20$, and $>20$ ) were assessed with the Cochran-Mantel-Haenszel test. Fisher's exact test was used to assess treatment differences in gender and race and in reason for discontinuation of study drug.

All efficacy analyses were performed on the intent-totreat (ITT) dataset, defined as all randomized patients who received study medication and who were rated on the PANSS total at baseline and at least once while receiving treatment. The primary efficacy variable was the change from baseline to the day 14 evaluation using the last observation carried forward (LOCF) for the PANSS total score. The day 14 evaluation point was chosen because it was thought that the 2-week acute stabilization period would be the most clinically relevant, and that this period would also be the most controlled environment in which to test for efficacy given the potential for variations in compliance and environmental factors after discharge. In addition, the day 14 evaluation point was also within the time period in which apparent efficacy was demonstrated in the previous Casey et al (2003) study, thus allowing possible comparison of efficacy between the two studies. Treatment group differences for the primary efficacy variable were assessed by a two-way ANOVA with factors for treatment group and study center. Based on the results of Casey et al (2003), the planned sample size of 200 subjects each in the antipsychotic monotherapy and combination therapy groups had $80 \%$ power to detect a treatment difference of 5.1 with a pooled standard deviation of 18.2 (standardized effect size $=0.28$ ) and a Type I error rate of 0.05 for a twotailed test.

Supportive efficacy endpoints included change from baseline to each scheduled visit (using LOCF) for the PANSS total, positive, and negative scores, the BPRS total score derived from the PANSS, and CGI severity and improvement scores. All secondary endpoints except the CGI Improvement score were assessed as described for the primary efficacy endpoint. For the CGI Improvement score, the primary treatment comparison was assessed by Cochran-Mantel-Haenszel with study centers as strata.

All randomized patients who received at least one dose of study medication were evaluated for safety. The primary treatment comparison for safety data was each individual combination therapy group $v s$ its corresponding antipsychotic monotherapy group (ie olanzapine/divalproex ER $v s$ olanzapine/placebo and risperidone/divalproex ER $v s$ risperidone/placebo). Adverse events were coded using the Coding Symbols for Thesaurus of Adverse Reaction Terms (COSTART) Version V dictionary. Treatment-emergent adverse events were those events that began or worsened in severity on or after the first day of dosing. Treatment differences in the percentage of patients reporting each treatment-emergent adverse event were assessed by Fisher's exact test. Treatment group differences in laboratory data were analyzed by a one-way ANOVA with treatment as the main effect. The primary analysis was the change from baseline (last determination obtained on or before day 1 of dosing) to the final evaluation for each variable.

\section{RESULTS}

A total of 402 patients were randomized for treatment: 103 received olanzapine/placebo, 99 received olanzapine/ divalproex ER, 101 received risperidone/placebo, and 99 received risperidone/divalproex ER. 251 patients (62\%) discontinued study drug. Reasons for premature discontinuations were adverse event (12\% monotherapy, $16 \%$ combination therapy), withdrew consent (21\% monotherapy, $17 \%$ combination therapy), lost to follow-up (5\% monotherapy, $7 \%$ combination therapy), noncompliance (7\% monotherapy, 9\% combination therapy), ineffectiveness (19\% monotherapy, $14 \%$ combination therapy), and other ( $12 \%$ monotherapy, $8 \%$ combination therapy); subjects may have reported more than one reason for premature discontinuations, but were counted only once in the total. No significant treatment differences were noted for overall discontinuation rate or discontinuation rate by specific reason. Overall, the majority of subjects who prematurely discontinued did so during the first 4 weeks of treatment $(37 \% ; 147 / 402)$. A total of $43,46,32$, and 30 patients in the olanzapine/placebo, olanzapine/divalproex $\mathrm{ER}$, risperidone/placebo, and risperidone/divalproex ER groups, respectively, completed the study.

Baseline demographic and clinical characteristics are listed in Table 1. The treatment groups did not differ in demographics, schizophrenia subtype, age at first diagnosis, or number of suicide attempts. Most patients $(86 \%)$ had a diagnosis of paranoid schizophrenia, with more than six lifetime hospitalizations (55\%), and at least one suicide attempt (51\%). The mean baseline PANSS total score was 99.1 for the antipsychotic monotherapy group and 98.3 for the combination therapy group.

\section{Efficacy Results}

Efficacy results are summarized in Figure 1 and Table 2. The primary efficacy variable, mean change from baseline to day 14 LOCF in PANSS total score, did not demonstrate a significant treatment difference between the combination therapy group and the monotherapy group $(p=0.307)$. Similarly, there was no treatment difference on the PANSS total at day $84(p=0.229)$. No statistically significant differences were found between treatment groups on the PANSS positive subscale at day $14(p=0.473)$ or day 84 $(p=0.623)$, or the PANSS general psychopathology subscale at day $14(p=0.603)$ or day $84(p=0.355)$. Results of the PANSS negative subscale demonstrated a numerical advantage of antipsychotic monotherapy over combination therapy $(p=0.085)$ at day 14 , and showed a significant treatment difference favoring antipsychotic monotherapy $v s$ combination therapy on days $3,5,7,10,42,49,63,77$, and 84 .

Change from baseline on the BPRSd was not significantly different for combination therapy $v s$ antipsychotic monotherapy at day $14(p=0.457)$ or at day $84(p=0.364)$. The CGI severity and improvement scores also failed to show a 
Table I Demographic Characteristics (Intent-to-Treat Dataset)

\begin{tabular}{|c|c|c|}
\hline $\begin{array}{l}\text { Demographic } \\
\text { characteristic }\end{array}$ & $\begin{array}{l}\text { Antipsychotic } \\
\text { monotherapy }\end{array}$ & $\begin{array}{c}\text { Combination } \\
\text { therapy }\end{array}$ \\
\hline Gender & $(N=198)$ & $(N=195)$ \\
\hline Female & $42(21 \%)$ & 47 (24\%) \\
\hline Male & 156 (79\%) & | 48 (76\%) \\
\hline Race & $(N=198)$ & $(N=195)$ \\
\hline White & $83(42 \%)$ & $93(48 \%)$ \\
\hline Black & $104(53 \%)$ & 95 (49\%) \\
\hline Other & || (5\%) & $7(3 \%)$ \\
\hline Age (years) & $(N=198)$ & $(N=195)$ \\
\hline Mean (SD) & $39.9(10.49)$ & $40.1(10.54)$ \\
\hline Range & $18-64$ & $18-65$ \\
\hline Weight (kg) & $(N=198)$ & $(N=195)$ \\
\hline Mean (SD) & $87.4(20.66)$ & $87.5(19.98)$ \\
\hline Range & $53.1-149.7$ & $53.5-148.8$ \\
\hline Schizophrenic subtype & $(N=198)$ & $(N=195)$ \\
\hline Paranoid type & $172(87 \%)$ & 166 (85\%) \\
\hline Disorganized type & $9(5 \%)$ & $7(4 \%)$ \\
\hline Catatonic type & $0(0 \%)$ & $\mid(<\mid \%)$ \\
\hline Undifferentiated type & $17(9 \%)$ & $21(11 \%)$ \\
\hline Age (years) at first diagnosis & $(N=195)$ & $(N=194)$ \\
\hline Mean (SD) & $23.8(8.1)$ & $24.6(8.5)$ \\
\hline Median & 22.0 & 22.0 \\
\hline $\begin{array}{l}\text { Lifetime number of } \\
\text { hospitalizations }\end{array}$ & $(N=198)$ & $(N=194)$ \\
\hline 0 & $3(2 \%)$ & $3(2 \%)$ \\
\hline $1-5$ & $76(38 \%)$ & $91(47 \%)$ \\
\hline $6-10$ & $52(26 \%)$ & $39(20 \%)$ \\
\hline$>10$ & $67(34 \%)$ & $61(31 \%)$ \\
\hline Number of suicide attempts & $(N=198)$ & $(N=195)$ \\
\hline 0 & 108 (55\%) & 85 (44\%) \\
\hline $\mid-5$ & $80(40 \%)$ & 99 (5।\%) \\
\hline$\geqslant 6$ & $10(5 \%)$ & II (6\%) \\
\hline
\end{tabular}

statistically significant difference between antipsychotic monotherapy and combination therapy at days 14 or 84 .

The use of adjunctive rescue medications (ie lorazepam, propranolol hydrochloride, and benztropine mesylate) during the study, including dosage (mg/day), number of days used, and percentage of patients using rescue medications, was similar between the antipsychotic monotherapy and combination therapy groups. Lorazepam was used by $65 \%$ of patients on antipsychotic monotherapy for an average of 6.2 days, and by $59 \%$ of patients on combination therapy for an average of 6.4 days. The mean daily lorazepam dose was $1.5 \mathrm{mg}$ in both groups. Only $16 \%$ of patients used either propranolol hydrochloride or benztropine mesylate.

\section{Safety Results}

The mean modal daily divalproex ER dose was $2828 \mathrm{mg}$ in the olanzapine/divalproex ER group and $2712 \mathrm{mg}$ in the risperidone/divalproex ER group. From weeks 2 through 12, the mean daily dose of olanzapine was $15 \mathrm{mg} / \mathrm{day}$, and the mean daily dose of risperidone was $6 \mathrm{mg} /$ day. According to pill counts, $82 \%$ of subjects were compliant at least $70 \%$ of the time. Valproate serum levels for observed cases are presented in Figure 2 and suggest a meaningful drop off in compliance following the inpatient phase of the study. The overall incidence of treatment-emergent adverse events was $83 \%$ in the olanzapine/placebo group, $88 \%$ in the olanzapine/divalproex ER group, $79 \%$ in the risperidone/placebo group, and $84 \%$ in the risperidone/divalproex ER group, with no significant differences between a specific combination therapy group and its corresponding antipsychotic monotherapy group. Most of the adverse events observed during the study were mild or moderate in severity. The incidence of constipation was significantly higher for risperidone compared to risperidone/divalproex ER (8 vs $1 \% ; p=0.035)$. Conversely, the incidence of somnolence ( 45 vs $19 \% ; p<0.001)$, weight gain (22 vs $10 \% ; p=0.021)$, and urinary incontinence ( 5 vs $0 \% ; p=0.028$ ) was significantly higher for risperidone/divalproex ER $v s$ risperidone monotherapy. The incidence of dry mouth was significantly higher in the olanzapine group compared to the olanzapine/ divalproex ER group (13 vs $4 \%$; $p=0.041$ ), but the incidence of back pain was significantly higher in the olanzapine/ divalproex ER group compared to the olanzapine monotherapy group (5 vs $0 \% ; p=0.027$ ). One patient in the risperidone/divalproex ER group died on day 24 due to an accidental opioid overdose, which was considered not related to study drug by the investigator.

Laboratory assessments showed a significant decrease from baseline in platelet counts in each combination therapy group when compared to its respective monotherapy group. The mean serum level of LDL cholesterol was statistically significantly decreased from baseline in the antipsychotic combination therapy group $v s$ the antipsychotic monotherapy group $(p=0.004)$. A similar decrease in LDL cholesterol level was seen when risperidone/divalproex ER was compared with risperidone monotherapy $(p=0.036)$ and when olanzapine/divalproex ER was compared with olanzapine monotherapy $(p=0.056)$. Table 3 presents the safety parameters by drug groups.

\section{DISCUSSION}

In this study, the addition of divalproex ER to olanzapine or risperidone did not enhance efficacy at either the primary endpoint of 14 days or in the extended 12-week treatment period. These results contrast somewhat with other reports of adjunctive or combination therapy using valproate in psychotic populations (eg Wassef et al, 1989, 2000) and with the findings from a similarly designed study of divalproex combination therapy with risperidone or olanzapine (Casey et al, 2003), in which improvement with divalproex was 


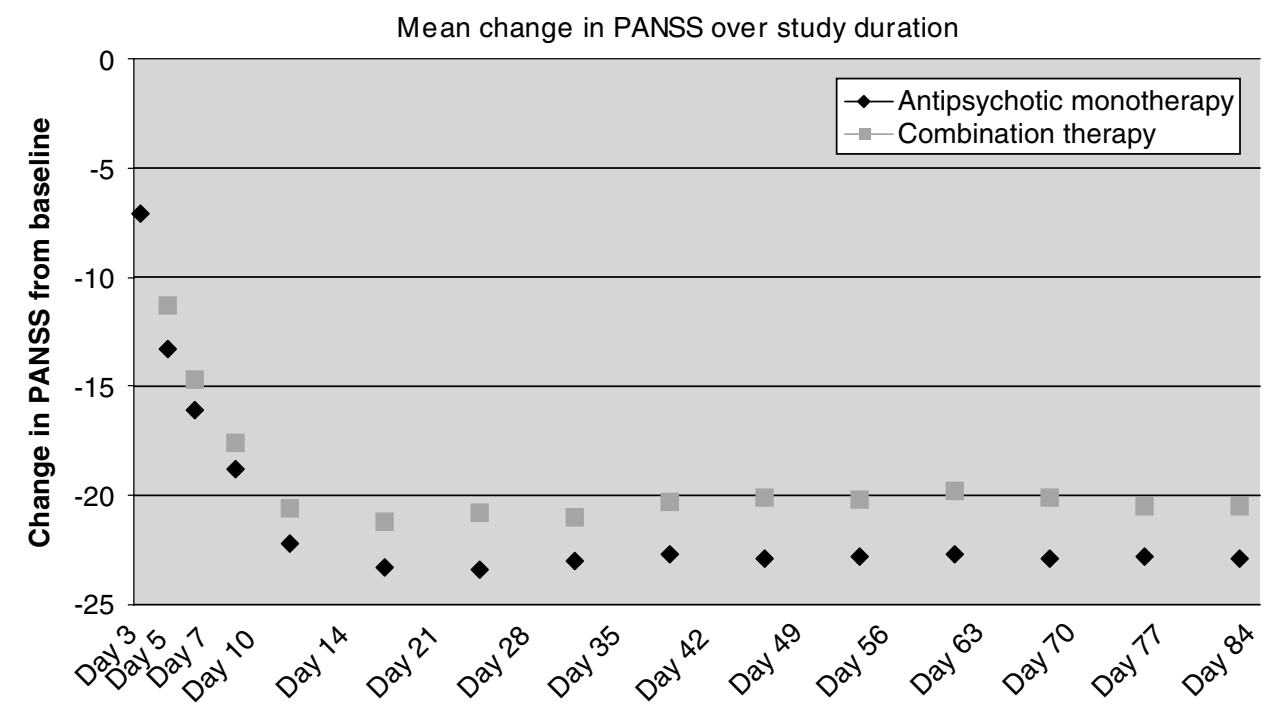

Figure I Change from baseline for the PANSS total LOCF. There were no significant differences between DVPX ER/antipsychotic combination therapy and antipsychotic monotherapy at any time point.

demonstrated as early as day 3 and which continued to day 21 , but which failed to reach its primary efficacy endpoint of PANSS change score from baseline to week 4. In this study, mean negative symptom scores on the PANSS Negative subscales improved from baseline in both groups, but more so in the monotherapy group. However, statistically significant treatment differences in negative symptoms favoring antipsychotic monotherapy $v s$ combination therapy were not seen in the earlier Casey et al (2003) study. It is possible that the effects of combination therapy on negative symptoms in this study were partially masked by sedative effects. For example, in this study, the incidence of somnolence was higher for the risperidone/divalproex ER $v s$ risperidone monotherapy group (45 vs $19 \%$; $p<0.001$ ).

Overall, the basis for the different outcomes in these two similar studies is unclear. A comparison between the Casey et al (2003) study and this study suggests similar patient characteristics at enrollment. The duration of this study was 12 weeks, compared to 4 weeks for the previous study, and the primary endpoint in this study was 14 days, compared to the 28-day primary endpoint in Casey et al (2003). Although the primary efficacy measure was the same in both studies, different supportive efficacy measures were used in this study. Another difference was that the Casey et al (2003) study utilized divalproex BID whereas this study utilized divalproex ER administered QD. To account for differences in bioavailability between formulations, this study used a more rapid titration schedule than Casey et al (2003). By day 14, dosing was comparable in the two studies, as were serum valproate concentrations (in $\mu \mathrm{g} / \mathrm{ml}$, OLZ + DVPX, mean (SD): 91.2 (33.04) (this study) vs 97.2 (28.47) (Casey et al, 2003); RISP + DVPX, mean (SD): 105.5 (27.26) (this study) vs 102.5 (24.15) (Casey et al, 2003); all DVPX, mean (SD): 97.9 (31.20) (this study) vs 99.7 (26.53) (Casey et al, 2003)).

Another difference between the two studies is the serum valproate levels of subjects in the later time points of the studies. Final day mean trough levels of valproate at day 28 in the Casey et al (2003) study were 98.2 and $100.2 \mu \mathrm{g} / \mathrm{ml}$ in combination with risperidone and olanzapine, respectively, compared to levels in the $65-80 \mu \mathrm{g} / \mathrm{ml}$ range at the later time points in this study. The decrease in serum valproate levels over time in this study may be due to a reduction in compliance during the outpatient portion of the study. As the therapeutic range for adjunctive divalproex in the treatment of schizophrenic symptoms is not well delineated, it is unclear if the serum valproate levels achieved after day 14 in this study should be adequate to treat the symptoms of schizophrenia; however, in a previously reported 4-week open-label study (Citrome et al, 2004), thirty schizophrenic subjects were converted from divalproex DR to ER, and the 12-h post-dose trough plasma levels were $80.1 \pm 20.4$ and $73.1 \pm 24.2 \mu \mathrm{g} / \mathrm{ml}$ (mean $\pm \mathrm{SD}$ ), respectively. In this previously reported study, psychopathology as measured on the total BPRS improved slightly $(p=0.0322)$, indicating that plasma levels of valproate in the vicinity of $73.1 \mu \mathrm{g} / \mathrm{ml}$ may have some therapeutic effect in schizophrenia. This suggests that the serum valproate levels achieved in the later stages of this study could be clinically relevant in the treatment of schizophrenia.

In addition, another key distinction between the two studies that is likely related to the differences in serum valproate levels discussed above is that in the Casey et al (2003) study, the subjects spent the study duration in an inpatient setting, whereas in this study, subjects could be discharged after 14 days of treatment. The length of time in the hospital very likely correlates with improved compliance, in contrast to an unsupervised outpatient setting. In Figure 1, the early reduction in psychotic symptoms in this study parallels the time course and degree of improvement that has been seen in several second-generation antipsychotic trials done primarily with inpatients (Casey et al, 2003). However, from days 14-84 there was virtually no change in response to 10 more weeks of treatment. This is highly unusual for antipsychotic trials in schizophrenia (Sherwood et al, 2006). Furthermore, the drop-off in serum valproate levels seen after day 14 (Figure 2) is consistent with decreased compliance as subjects left the inpatient 
Table 2 Baseline and Mean Change from Baseline to Days 14 and 84 LOCF for Efficacy Variables (Intent-to-Treat Dataset)

\begin{tabular}{cccc}
\hline Efficacy & $\begin{array}{c}\text { Antipsychotic } \\
\text { monotherapy } \\
\text { mean (SE) } \\
\text { variable }\end{array}$ & $\begin{array}{c}\text { Combination } \\
\text { therapy } \\
\text { mean (SE) } \\
\mathbf{N}=\mathbf{I 9 5}^{\mathbf{a}}\end{array}$ & $\mathbf{P}$ \\
\hline PANSS total & & & \\
Baseline & $99.1(1.00)$ & $98.3(1.03)$ & $0.49 \mid$ \\
Day 14 & $-22.2(1.33)$ & $-20.6(1.37)$ & 0.307 \\
Day 84 & $-22.9(1.59)$ & $-20.5(1.64)$ & 0.229
\end{tabular}

$\begin{array}{llll}\text { PANSS positive } & & & \\ \text { Baseline } & 26.7(0.33) & 26.7(0.34) & 0.989 \\ \text { Day 14 } & -7.3(0.42) & -6.9(0.43) & 0.473 \\ \text { Day 84 } & -7.5(0.50) & -7.2(0.52) & 0.623\end{array}$

PANSS negative

$\begin{array}{llll}\text { Baseline } & 24.4(0.40) & 23.8(0.41) & 0.169 \\ \text { Day 14 } & -4.8(0.40) & -3.9(0.41) & 0.085 \\ \text { Day 84 } & -4.9(0.44) & -3.9(0.45) & 0.045\end{array}$

PANSS general psychopathology

$\begin{array}{lrrl}\text { Baseline } & 47.9(0.54) & 47.8(0.56) & 0.811 \\ \text { Day 14 } & -10.2(0.71) & -9.7(0.73) & 0.603 \\ \text { Day 84 } & -10.5(0.84) & -9.5(0.87) & 0.355\end{array}$

BPRSd

$\begin{array}{lrrr}\text { Baseline } & 58.1(0.57) & 58.1(0.59) & 0.976 \\ \text { Day I4 } & -13.9(0.82) & -13.2(0.84) & 0.457 \\ \text { Day 84 } & -14.3(0.97) & -13.2(1.00) & 0.364\end{array}$

$\begin{array}{crcc}\text { CGI severity } & & & \\ \text { Baseline } & 5.0(0.05) & 5.0(0.5) & 0.633 \\ \text { Day 14 } & -1.0(0.07) & -1.1(0.07) & 0.872 \\ \text { Day 84 } & -1.2(0.08) & -1.1(0.08) & 0.725\end{array}$

CGI improvement

$\begin{array}{llll}\text { Day 14 } & 2.6(0.08) & 2.7(0.08) & 0.362 \\ \text { Day } 84 & 2.7(0.10) & 2.7(0.09) & 0.720\end{array}$

${ }^{\mathrm{a}} \mathrm{N}=194$ for $\mathrm{CGl}$ severity.

setting. Additionally, the percent of patients completing this study was lower than usual for antipsychotic trials, and was likely influenced by the lengthy period of time that many patients spent as outpatients, during which many extraneous variables may have influenced their participation and/or compliance in the study.

An examination of the efficacy results from the current trial and the similarly designed Casey et al (2003) study suggests that the combination therapies led to similar improvements through day 14 (the primary endpoint in this study). Figure 3 illustrates the change from baseline on PANSS total at day 14 for antipsychotic monotherapy and combination therapy for both studies. The striking differ-

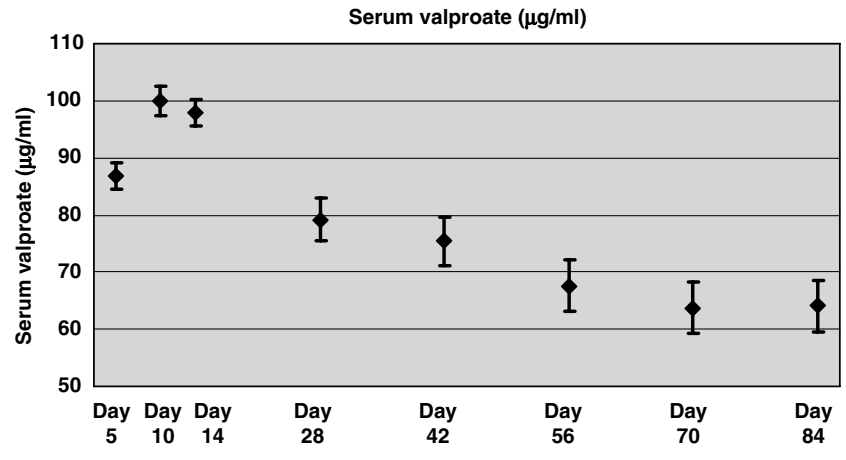

Figure 2 Serum trough valproate levels $(\mu \mathrm{g} / \mathrm{ml})$ mean and SE by Study Day for observed cases. The drop in serum levels on day 14 (after the inpatient phase) suggests decreased compliance in the outpatient phase of the study.

ence between the studies is the change from baseline for the antipsychotic monotherapy groups. In fact, the difference in change scores for antipsychotic monotherapy across studies is larger than the within-study change scores between the antipsychotic monotherapy and combination therapy groups that led to a statistically significant result in Casey et al (2003). Likewise, the responder rates (20\% improvement) across studies show that the most profound difference from one study to the next was in the antipsychotic monotherapy group. The combination therapy responder rates at day 14 were similar between the Casey et al (2003) study and this study (66 and 65\%, respectively); however, the rate of responders in the antipsychotic monotherapy group was $53 \%$ in Casey et al (2003) vs $71 \%$ in this study. Taken together, these data suggest the possibility of a greater placebo response in this study, which may have diminished the apparent efficacy of the combination treatment when compared against the monotherapy treatment.

This study did not raise new safety concerns; the addition of divalproex ER to olanzapine or risperidone was associated with known adverse events such as somnolence, weight gain, and decreased platelet count. Consistent with a number of other previous studies across a variety of illnesses (Swann et al, 2001; Jafari et al, 2002; Beydoun et al, 2002; Bowden et al, 2006), divalproex ER lowered serum total and LDL cholesterol. This study extended previous findings showing that divalproex mitigates the increase in total cholesterol associated with olanzapine and risperidone (Jafari et al, 2003), an effect that remained robust through 12 weeks of combination therapy. Although not statistically significant, it is noteworthy that divalproex ER appeared to lower serum HDL cholesterol in the combination treatment group compared to the antipsychotic monotherapy group.

In conclusion, the lack of a significant efficacy benefit from adding divalproex ER to risperidone or olanzapine in this study contrasts with previous reports of efficacy for valproate combination therapy. Considering the potential for increased safety risks associated with combination treatment and the conflicting data regarding its efficacy, the use of antipsychotic/divalproex ER combination treatment for the treatment of schizophrenia is not strongly supported. Indeed, further studies are required to clarify the 
Table 3 Mean Change from Baseline to Final Evaluation for Safety Parameters of Special Interest

\begin{tabular}{|c|c|c|c|c|}
\hline Measure & OLZ $^{\mathrm{a}}$ & OLZ+DVPX ${ }^{a}$ & $\mathbf{R I S P}^{\mathbf{a}}$ & RISP+DVPX ${ }^{a}$ \\
\hline \multicolumn{5}{|c|}{ Platelet count $\left(\times 10^{9} / \mathrm{L}\right)$} \\
\hline Baseline & 255.06 & 248.14 & 252.23 & 254.94 \\
\hline Change (SD) & $-3.32(49.15)$ & $-42.34(45.32)^{b}$ & $-3.02(39.16)$ & $-55.43(65.24)^{b}$ \\
\hline \multicolumn{5}{|l|}{ SGOTIAST (IUIL) } \\
\hline Change (SD) & $3.88(14.08)$ & $5.14(16.48)$ & $1.82(16.13)$ & $0.96(12.36)$ \\
\hline \multicolumn{5}{|l|}{ SGPT/ALT (IU/L) } \\
\hline Baseline & 23.58 & 21.73 & 19.88 & 22.73 \\
\hline Change (SD) & 7.73 (28.77) & 7.74 (29.95) & $4.78(29.33)$ & $-1.13(20.18)$ \\
\hline \multicolumn{5}{|l|}{ Glucose } \\
\hline \multicolumn{5}{|l|}{ Triglycerides } \\
\hline Baseline & 172.55 & 184.54 & 155.50 & 155.39 \\
\hline Change (SD) & 9.38 (95.02) & I $6.27(\mid 58.01)$ & $-12.18(92.47)$ & | 4.87 (I54.24) \\
\hline \multicolumn{5}{|l|}{ Total cholesterol } \\
\hline Baseline & 198.36 & 189.06 & 190.68 & 193.00 \\
\hline Change (SD) & 7.00 (35.7I) & $5.36(34.72)$ & 1.32 (33.99) & $-5.49(40.09)$ \\
\hline \multicolumn{5}{|l|}{ HDL cholesterol } \\
\hline \multicolumn{5}{|l|}{ Wt (kg) } \\
\hline Baseline & 88.1 & 86.7 & 86.6 & 89.0 \\
\hline Change (SD) & $2.8(4.38)$ & $4.5(4.65)^{b}$ & $2.0(3.76)$ & $3.3(4.54)^{b}$ \\
\hline
\end{tabular}

aSample sizes range from 94 to I0I for OLZ, 87-99 for OLZ+DVPX, 95-10I for RISP, and from 92 to 99 for RISP+DVPX.

${ }^{b}$ Comparison with monotherapy significant at $p<0.05$.

${ }^{c}$ Comparison with monotherapy $p=0.056$.

Across-Study Comparison of Efficacy

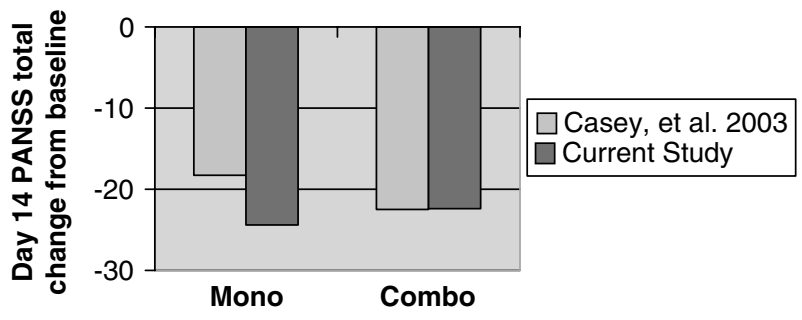

Figure 3 Across-study comparison of efficacy at day 14. The combination therapy groups across the two studies (this study and Casey et al, 2003) had identical PANSS total changes at day 14, however, the monotherapy group showed greater improvement in this study. conflicting efficacy results reported to date and to better define the risk/benefit ratio of adding divalproex ER to olanzapine or risperidone in the treatment of acute schizophrenia.

\section{ACKNOWLEDGEMENTS}

This study was supported by Abbott Laboratories, Abbott Park, IL. We thank Sharon Stec of Abbott Laboratories who served capably as clinical project manager for this study. We also thank the members of the Depakote ER Combination Treatment Study Group, as follows, for their enrollment of patients and participation in this study: Jose J 
Alvarez, MD, Comprehensive NeuroScience Inc., Melbourne, FL; Mohammed Bari, MD, Synergy Clinical Research, Chula Vista, CA; Louise M Beckett MD, IPS Research Company, Oklahoma City, OK; Jeffrey A Borenstein, MD, Holliswood Hospital, Holliswood, NY; Guy E Brannon, MD, Brentwood Research Institute, Shreveport, LA; David W Brown, MD, Community Clinical Research Inc., Austin, TX; Jesse M Carr, MD California Clinical Trials Medical Group Inc., Glendale, CA; Lori L Davis, MD, Tuscaloosa VA Medical Center, Tuscaloosa, AL; David Feifel, MD, PhD, UCSD Medical Center, San Diego, CA; David H Flaherty, DO, Segal Institute for Clinical Research, North Miami, FL; Donald J Garcia, Jr, MD, Future Search Trials, Austin, TX; Lev Gertsik, MD, Cedars Sinai Medical Center, Los Angeles, CA; Steven Glass, MD, CNS Research Institute, PC, Clementon, NJ; Armen Goenjian, MD, Collaborative NeuroScience Network Inc., Garden Grove, CA; Clifford Goldman, MD, ClinSearch Inc., Kenilworth, NJ; Ram Gopalan, MD, Comprehensive Neuroscience of Northern Virginia, Falls Church, VA; Richard R Jaffe, MD, Belmont Center for Comprehensive Treatment, Philadelphia, PA; John Kasckow, MD, PhD, University of Cincinnati Medical Center, Cincinnati, OH; Richard D Knapp, DO, CORE Research Inc., Winter Park, FL; Mary Ann Knesevich, MD, University Hills Clinical Research, Irving, TX; Joseph Kwentus, MD, University of Mississippi Medical Center, Jackson, MS; Mark N Lerman, MD, Comprehensive NeuroScience Inc., Hoffman Estates, IL; Robert E Litman, MD, Centers for Behavioral Health, LLC, Rockville, MD; H Edward Logue, MD, Birmingham Psychiatry Pharmaceutical Studies Inc., Birmingham, AL; Joseph P McEvoy, MD, Duke University Medical Center, Butner, NC; Alexander L Miller, MD, University of Texas Health Science Center at San Antonio, San Antonio, TX; Michael G Plopper, MD, Sharp Mesa Vista Hospital, San Diego, CA; Raj Rajani, MD, Behavioral and Medical Research, LLC, Anaheim, CA; Robert A Riesenberg, MD, Atlanta Center for Medical Research, Atlanta, GA; Murray H Rosenthal, DO, HealthQuest Clinical Trials, San Diego, CA; David Sack, MD, Comprehensive NeuroScience Inc., Cerritos, CA; Scott D Segal, MD, Segal Institute for Clinical Research, North Miami, FL; Rajiv P Sharma, MD, The Psychiatric Institute University of Illinois at Chicago Medical Center, Chicago, IL; Anantha Shekhar, $\mathrm{MD}, \mathrm{PhD}$, Indiana University, Indianapolis, IN.

\section{DISCLOSURES}

Daniel Casey has received consultant fees from Abbott Laboratories, Bristol-Myers Squibb, Dainippon Sumitomo Pharmaceuticals, Janssen Pharmaceuticals, NuPathe Inc., Pfizer Inc., Solvay Pharmaceuticals and Wyeth Pharmaceuticals. He has been on Speakers Bureaus for Abbott Laboratories, Bristol-Meyers Squibb, Janssen Pharmaceuticals and Pfizer. Dr Casey receives financial support from Danicas Foundation. John Kane has served as a consultant for Abbott Laboratories, Astra-Zeneca, Bristol-Meyers Squibb, Janssen, Eli Lilly and Company, Pfizer Inc., Wyeth Pharmaceuticals and on Speakers Bureaus for BristolMeyers Squibb, Janssen, and Astra-Zeneca. Carol Tamminga has been a paid consultant for Acadia Pharmaceuticals,
Intra-cellular Therapies, Orexigen, Alexza Pharmaceuticals, and Lunbeck Inc. She is on the Advisory Boards for Acadia Pharmaceutical and Intracellular Therapies. David Daniel is employed by United Biosource Corporation. Walid AbiSaab and Patricia Wozniak are former employees of Abbott Laboratories. Laura Redden, Mario Saltarelli, Jeff Baker and Nicholas Greco are current employees of and owners of equity in Abbott Laboratories. Tram Tran-Johnson was an investigator in this study and reports no other financial conflicts.

\section{REFERENCES}

Barnes TR (1989). A rating scale for drug-induced akathisia. $\mathrm{Br} J$ Pharmacol 154: 672-676.

Benes FM (2000). Emerging principle of altered neural circuitry in schizophrenia. Brain Res Brain Res Rev 31: 251-269.

Berle JO, Spigset O (2005). Are mood stabilizers beneficial in the treatment of schizophrenia? Tidsskr Nor Laegeforen 125: 1809-1812.

Beydoun A, Jafari M, Wozniak P, Giordano S, Sommerville K (2002). Cholesterol-lowering effect of divalproex sodium is concentration-related in a monotherapy study of complex partial seizures. In: Program and Abstracts of the 2002 Annual Meeting of the American Epilepsy Society. Seattle, WA, December 2002.

Bowden CL, Swann AC, Calabrese JR, Rubenfaer LM, Wozniak PJ, Collins MA et al (2006). A randomized, placebo-controlled, multi-center study of divalproex sodium extended-release in the treatment of acute mania. J Clin Psychiatry 67: 1501-1510.

Casey DE, Daniel DG, Wassef AA, Tracy KA, Wozniak P, Sommerville KW (2003). Effect of divalproex combined with olanzapine or risperidone in patients with an acute exacerbation of schizophrenia. Neuropsychopharmacology 28: 182-192.

Chong SA, Tan CH, Lee L, Liow PH (1998). Augmentation of risperidone with valproic acid [letter]. J Clin Psychiatry 59: 430.

Citrome L, Tremeau F, Wynn PS, Roy B, Dinakar H (2004). A study of the safety, efficacy, and tolerability of switching from the standard delayed release preparation of divalproex sodium to the extended release formulation in patients with schizophrenia. J Clin Psychopharmacol 24: 255-259.

First MB, Spitzer RL, Gibbon M, Williams JBW (2002). Research Version of the Structured Clinical Interview (SCID) for DSM-IVTR Axis 1 Disorders, Modified for Abbott Laboratories Protocol No. M02-547. New York State Psychiatric Institute, New York, New York.

Gunderewa VM, Beckman H, Zimmer R, Ruther E (1980). Effect of valproic acid on schizophrenic syndromes. Arzneimittelforschung 30: 1212-1213.

Guy W (ed) (1976). ECDEU Assessment Manual for Psychopharmacology, publication No. ADM 76-336. Rockville, MD, US Department of Health, Education, and Welfare.

Jafari M, Jiang P, Casey DE (2003). Divalproex adjunctive therapy lowers elevated cholesterol associated with olanzapine and risperidone treatment of schizophrenia. Paper presented to International Congress on Schizophrenia research, 2003.

Jafari M, Willmore LJ, Wozniak P, Giordano S, Sommerville K (2002). Cholesterol-lowering effects of divalproex sodium in adult patients with complex partial seizure. In: Program and Abstracts of the 2002 Annual Meeting of the American Epilepsy Society. Seattle, WA, December 2002.

Kay SR, Fiszbein A, Opler LA (1987). The positive and negative syndrome scale (PANSS) for schizophrenia. Schizophr Bull 13: 261-276.

Lewis DA, Hashimoto T, Volk DW (2005). Cortical inhibitory neurons and schizophrenia. Nat Rev Neurosci 6: 312-324. 
Meltzer HY (1999). Outcome in schizophrenia: beyond symptom reduction. J Clin Psychiatry 60(Suppl. 3): 3-7.

Melzer HY, Dai J, Ichikawa J (2001). Valproic acid, an anticonvulsant mood stabilizer, potentiates antipsychotic-induced dopamine release in rat medial prefrontal cortex but not the nucleus accumbens. Society for Neuroscience Meeting: San Diego. (Abstract).

Möller HJ (2003). Management of negative symptoms of schizophrenia: new treatment options. CNS Drugs 17: 793-823.

Moringo A, Martin J, Gonzales S, Mateo I (1989). Treatment of resistant schizophrenia with valproate and neuroleptic drugs. Hillside J Clin Psychiatry 11: 199-207.

Sherwood M, Thornton AE, Honer WG (2006). A meta-analysis of profile and time-course symptom change in acute schizophrenia treated with atypical antipsychotics. Int J Neuropsychopharmacol 9: 357-366.
Simpson G, Angus JSW (1970). A rating scale for extrapyramidal side effects. Acta Psychiatr Scand 212: 11-19.

Swann A, Sommerville K, Tracy K, Wozniak P, Jafari M, Collins M (2001). Lack of metabolic effects of short-term divalproex sodium treatment. In: Program and Abstracts of the 2001 Annual Meeting of the American College of Neuropsychopharmacology. Puerto Rico, December 2001.

Wassef A, Baker J, Kochan LD (2003). GABA and schizophrenia: a review of basic science and clinical studies. J Clin Psychopharmacol 23: 601-640.

Wassef A, Watson DJ, Morrison P, Bryant S, Flack J (1989). Neuroleptic-valproic acid combination in treatment of psychotic symptoms: a three-case report. J Clin Psychopharmacol 9: 45-48.

Wassef AA, Dott SG, Harris A, Brown A, O’Boyle M, Meyer III WJ (2000). Randomized, placebo-controlled pilot study of divalproex sodium in the treatment of acute exacerbations of chronic schizophrenia. J Clin Psychopharmacol 20: 357-361. 